\title{
ASPECTOS MORFOLÓGICOS DE Annona squamosa L. (Annonaceae) SOB INFLUÊNCIA DE DIFERENTES PROCESSOS DE SUPERAÇÃO DE DORMÊNCIA
}

Bruno Araújo de Souza ${ }^{1}$, Igor Araújo de Souza1, Oriales Rocha Pereira ${ }^{2}$

${ }^{1}$ Acadêmicos do curso de Ciências Biológicas, Universidade do Estado de Mato

Grosso, Campus de Nova Xavantina (bruno.araujo10@hotmail.com)

2 Professora Mestre da Faculdade de Ciências Agrárias, Biológicas e Sociais

Aplicadas, Universidade do Estado de Mato Grosso

Recebido em: 06/04/2018 - Aprovado em: 10/06/2018 - Publicado em: 20/06/2018

DOI: 10.18677/EnciBio_2018A10

\begin{abstract}
RESUMO
Annona squamosa L. possui um alto potencial econômico, no entanto já é conhecido que esta espécie possui dormência em suas sementes, necessitando de condições ambientais favoráveis para a germinação e desenvolvimento. Este estudo tem por objetivo verificar as diferenças no desenvolvimento dos órgãos vegetativos de indivíduos jovens que foram submetidos a diferentes processos de superação de dormência. $O$ trabalho foi conduzido em casa de vegetação da UNEMAT, Campus de Nova Xavantina, onde o tratamento 1- controle, 2- imersão em água durante 6 horas, 3- imersão em água por 24 horas, 4- escarificação mecânica e 5- imersão em ácido sulfúrico. Após 60 dias do plantio foram coletadas aleatoriamente sete plantas de cada tratamento, e em seguida com auxílio de um paquímetro foram realizadas as métricas morfológicas: tamanho: ápice da raiz ao ápice caulinar, quantidade de folhas de cada indivíduo, comprimento e largura do limbo foliar. Os resultados mostram, a partir das análises estatísticas, que houve diferença significativa para tamanho dos indivíduos jovens (TIJ) dentre todos os tratamentos, com as maiores métricas obtidas em indivíduos do tratamento quatro (escarificação mecânica), para a quantidade de folhas o tratamento três se sobressaiu entre os tratamentos, porém o teste não se mostrou significativo. O maior crescimento dos indivíduos pode estar relacionado com a exposição do embrião aos fatores externos, ativando antecipadamente as suas funções fisiológicas, que se desenvolveu primeiro, quando comparado aos embriões dos demais tratamentos. Estudos para conhecer a melhor forma de produção de mudas com potencial econômico são fundamentais para auxiliar os pequenos produtores no cultivo de diversas espécies.
\end{abstract}

PALAVRAS - CHAVE: condições ambientais, desenvolvimento inicial; in natura.

\section{MORPHOLOGICAL ASPECTS OF Annona squamosa L. (Annonaceae) UNDER INFLUENCE OF DIFFERENT DENTAL OVERCOMING PROCESSES}

\begin{abstract}
Annona squamosa L. has a high economic potential, however it is already known that this species has dormancy in its seeds, necessitating favorable environmental conditions for germination and development. This study aims to verify the differences in the development of the vegetative organs of young individuals who were submitted to different processes of dormancy overcoming. The work was conducted in a
\end{abstract}


greenhouse of UNEMAT, Campus of Nova Xavantina, where the treatment 1-control, 2-immersion in water for 6 hours, 3-immersion in water for 24 hours, 4-mechanical scarification and 5 -immersion in sulfuric acid. After 60 days of planting, seven plants were randomly collected from each treatment, and then, with the aid of a pachymeter, the morphological metrics were performed: size: apex of the root to the apex of the cauline, number of leaves of each individual, length and width of the leaf blade. The results show, from the statistical analyzes, that there was a significant difference in the size of the young individuals (SYI) among all treatments, and the highest metrics was obtained in individuals from the treatment four (mechanical scarification); about the number of leaves, the treatment three stood out among the treatments, although the test was not significant. The higher growth of the individuals may be related to the exposure of the embryo to the external factors, activating in advance their physiological functions, which developed first when compared to the embryos of the other treatments. Studies to know the best way of producing seedlings with economic potential are fundamental to assist small producers in the cultivation of several species.

KEYWORDS: environmental conditions, initial development, in natura.

\section{INTRODUÇÃO}

As anonáceas são representadas por um grande número de gêneros e espécies, a maior parte delas é oriunda de regiões tropicais ou subtropicais (NETO et al., 2014). A Annona squamosa L. popularmente conhecida como ata, pinha ou fruta-do-conde é destaque no comércio de fruto fresco, sendo muito utilizada para o consumo in natura (LEMOS, 2014; BATAGLION et al., 2015).

A reprodução sexuada dessa espécie e sua dispersão geralmente ocorrem por meio de sementes, mas a reprodução também acontece de forma assexuada e antrópica, onde essa propagação é realizada, por exemplo, por meio do método de enxertia, utilizado na reprodução de clones férteis (FERREIRA et al., 2002), pois um fator que pode ser limitante ao desenvolvimento de $A$. squamosa L. é a polinização, devido ser uma espécie que possui um sistema reprodutivo do tipo alógama/misto, predominantemente fecundação cruzada mas podendo ocorrer autofecundação (RESENDE, 2002), que está associado ao auxílio de polinizadores naturais, como os coleópteros para a família Annonaceae, no entanto a abundância destes artrópodes está diretamente ligada com condições do ambiente, assim como as taxas de frutificação da espécie, o que atualmente é influenciada de forma negativa, por exemplo pela ação de agentes ambientais como: ventos e precipitação (SÁNCHEZ, 1991).

As sementes produzidas por $A$. squamosa durante o processo de reprodução possuem estágio de dormência, resultando em inibição da germinação após a secagem da semente. A dormência é um processo fundamental para a sobrevivência do embrião no interior da semente. É uma estratégia de assegurar que as condições estejam favoráveis para o crescimento da plântula quando ocorrer a germinação, assim, constitui um evento fisiológico natural de resistência a mecanismos/fatores externos imposto pelo meio (TAIZ; ZEIGER, 2013; RAVEN et al., 2014).

Essa dormência pode ser ocasionada pela imaturidade fisiológica do embrião ou impermeabilidade e resistência do tegumento da semente. Grande parte das plantas nativas possui dormência no tegumento, diminuindo assim a entrada de água e oxigênio (RAVEN et al., 2014). Tal fato impede o crescimento do embrião, 
pois é uma resistência física intrínseca desse tipo de semente, e que se torna um fator físico a ser transposto (MAYAER; POLJAKOFF-MAYBER, 1982).

Muitos trabalhos vêm sendo realizados no intuito de se verificar os diferentes métodos de superação de dormência e a produção de mudas de árvores frutíferas para a comercialização: Neto et al., (2013) em seu trabalho avaliaram a taxa de germinação e o vigor das sementes de Annona squamosa L. de cinco localidades , onde foram submetidas a imersão em ácido giberélico $\left(\mathrm{GA}_{3}\right)\left(750 \mathrm{mg} \mathrm{L}^{-1}\right)$ por $12 \mathrm{~h}$ para superação da dormência. Esses autores consideraram a taxa de germinação, a velocidade de germinação e a quantidade de sementes duras e mortas, e os resultados mostraram que as procedências metodológicas influenciam significativamente no vigor e confere maior desempenho germinativo para a espécie. Estes resultados corroboram os dados encontrados por Stenzel et al., (2003), onde os autores compararam a superação de dormência de atemóia (Annona cherimola Mill.) e da pinha (Annona squamosa L.), ambas representantes da família Annonaceae.

Tratamentos pré-germinativos mecânicos e não mecânicos tais como escarificação mecânica e química, embebição em água, entre outros (ATAÍDE et al., 2013; FREITAS et al., 2013; SANTOS et al., 2014; CAMPOS et al., 2015; BAGATIM et al., 2016), podem provocar alteração na temperatura, permeabilidade dos gases, remoção de inibidores ou de promotores da germinação, influenciando no metabolismo e, portanto na dormência das sementes (SILVA, 2003).

Após a superação da dormência e durante a germinação do embrião, os tecidos meristemáticos apicais estão envolvidos diretamente no crescimento do corpo da planta, contribuindo, assim, para a formação dos órgãos vegetativos raiz, caule e folha, e o estabelecimento desses tecidos podem ser influenciados pelos métodos de superação de dormência, o que pode resultar em alterações morfológicas nos indivíduos jovens da espécie (RAVEN et al., 2014).

Estudos buscando entender essas influências ainda são escassos, assim, este trabalho teve por objetivo, verificar as diferenças no desenvolvimento morfológico dos órgãos vegetativos de Anonna squamosa L., analisando tamanho da planta jovem, da raiz ao ápice; quantidade de folhas e; comprimento e largura do limbo foliar de indivíduos jovens dessa espécie, provenientes de embriões de sementes que foram submetidas a cinco tratamentos de superação de dormência.

\section{MATERIAL E MÉTODOS}

O presente trabalho foi desenvolvido no Viveiro Florestal da Universidade do estado de Mato Grosso, Campus de Nova Xavantina- MT, localizado nas coordenadas $\left(14^{\circ} 41^{\prime} 20^{\prime \prime S}\right.$ e $\left.52^{\circ} 20^{\prime} 55^{\prime \prime} \mathrm{W}\right)$.

As sementes de Annona squamosa L. foram submetidas a cinco métodos de tratamentos para a superação de dormência: a) tratamento 1 - controle (sem nenhum método ativo para a superação de dormência); b) tratamento 2 - embebição em água durante 6 horas; c) - tratamento 3 - embebição em água durante 24 horas; d) tratamento 4 - escarificação mecânica e; e) tratamento 5 - imersão em ácido sulfúrico. Esses métodos foram adotados para superação de dormência tegumentar das sementes dessa espécie, e foram adaptados seguindo as metodologias de Piveta et al., (2014) e Bezerra (2015).

Após a exposição das sementes aos tratamentos supra citados, elas foram colocadas para germinar em tubetes, o que aconteceu em 08/09/2015. Cada tubete recebeu três sementes. Esses tubetes foram dispostos em bandejas de acordo com o tratamento específico de cada semente. O processo de germinação e crescimento 
ocorreu em casa de vegetação coberta com sombrite (50\% de luz) e irrigação intermitente de 12 em 12 horas.

Após 60 dias do plantio, foram escolhidos aleatoriamente, sete indivíduos de cada tratamento, para a caracterização morfológica. Foi observado e medido com auxílio de um Paquímetro (Mitutoyo) o tamanho da planta, sendo essas medidas mensuradas do ápice da raiz ao ápice caulinar. Também foi verificada a quantidade de folhas de cada indivíduo, comprimento das folhas e largura do limbo foliar (metodologia adaptada de PIMENTA et al., 2013).

A avaliação da influência dos diferentes métodos de superação de dormência sobre a estrutura morfológica dos indivíduos foi realizada por meio de estatística comparativa das médias de cada caráter analisado, com Análise de Variância ANOVA, através do teste de Tukey a $5 \%$ de probabilidade para comprimento do limbo foliar (CF), largura da folha (LF) e tamanho da planta (TP), e teste de KruskalWallis a $5 \%$ de probabilidade para a variável quantidade de folhas (QF), pois essa característica não atendeu ao pressuposto normalidade. A normalidade dos dados foi verificada através do teste Shapiro-Wilk (ZAR, 2010). Os dados coletados foram tabulados usando o Microsoft Excel e para as análises estatísticas foi utilizado o programa ASSISTAT Versão 7.7 beta (2016) (SILVA, 2016).

\section{RESULTADOS E DISCUSSÃO}

$\mathrm{Na}$ avaliação do desenvolvimento morfológico dos indivíduos de Annona squamosa L., em resposta aos diferentes métodos de superação de dormência que as sementes foram submetidas, os indivíduos apresentaram diferenças significativas para o caráter tamanho da planta (TP), com indivíduos do tratamento 4, escarificação mecânica, apresentando os maiores tamanhos, 24, $85 \mathrm{~cm}$ (Tabela 1).

Para o comprimento do limbo foliar (CF) e largura do limbo foliar (LF) as médias analisadas não diferiram estatisticamente entre os tratamentos. Quanto a quantidade de folhas (QF), o tratamento 3 apresentou maior média (23,64 folhas), porém não significativo estatisticamente (Tabela1).

TABELA 1. Análise comparativa das médias dos indivíduos entre os tratamentos. Sendo: comprimento da folha (CF), largura do limbo foliar (LF), quantidade de folhas (QF) e tamanho da planta (TP).

\begin{tabular}{ccccc}
\hline Tratamento & CF & LF & QF & TP \\
\hline $\mathbf{1}$ & $3,29 \mathrm{a}$ & $1,54 \mathrm{a}$ & $14,64 \mathrm{a}$ & $19,71 \mathrm{bc}$ \\
$\mathbf{2}$ & $3,71 \mathrm{a}$ & $1,88 \mathrm{a}$ & $18,92 \mathrm{a}$ & $21,57 \mathrm{~b}$ \\
$\mathbf{3}$ & $3,75 \mathrm{a}$ & $1,79 \mathrm{a}$ & $23,64 \mathrm{a}$ & $21,78 \mathrm{~b}$ \\
$\mathbf{4}$ & $3,99 \mathrm{a}$ & $1,90 \mathrm{a}$ & $18,92 \mathrm{a}$ & $24,85 \mathrm{a}$ \\
$\mathbf{5}$ & $3,26 \mathrm{a}$ & $1,58 \mathrm{a}$ & $13,85 \mathrm{a}$ & $17,57 \mathrm{c}$ \\
Valor de $\boldsymbol{p}$ & $\mathbf{0 , 1 3}$ & $\mathbf{0 , 1 0}$ & $>\mathbf{0 , 0 5}$ & $\mathbf{0 , 0 0 0 4}$ \\
\hline
\end{tabular}

Os valores seguidos pela mesma letra não diferem estatisticamente entre si.

A rápida emergência de plântulas e estabelecimento de plantas favorece o desenvolvimento dos órgãos vegetativos, e isso ocorre em sementes com embriões que conseguem mobilizar em menos tempo as reservas energéticas, tanto de cotilédones quanto de endosperma, proporcionando maior crescimento inicial e desenvolvimento (VIERA; CARVALHO, 1994). 
Após 60 dias a partir da data de semeadura foi notável o crescimento inicial das plantas, que apresentaram ganhos em altura e diâmetro do caule, estando fortemente relacionado com a absorção de água, nutrientes, gás carbônico e luz solar, pois segundo Azevedo et al. (2012), a absorção de água e nutrientes, juntamente com a presença de gás carbônico e exposição à luz, permite que os processos de fotossíntese aconteçam nos vegetais, o que proporciona o crescimento.

O maior crescimento ocorreu para os indivíduos procedentes do método de escarificação mecânica, os quais foram os primeiros que germinaram e também apresentaram os maiores tamanhos de limbo foliar. É nas células do limbo foliar que se localiza o aparato fotossintético da planta e onde ocorre o processo de fotossíntese (RAVEN, 2014). É através da eficiência fotossintética que ocorre a alocação de matéria orgânica para o maior desenvolvimento estrutural do vegetal, e parte dessa matéria possibilita o crescimento oriundo de atividades de meristemas apicais (TAIZ; ZEIGER, 2013).

Embora o resultado de CF, LF e QF não apresentaram diferença significativa entre os tratamentos, o presente estudo evidenciou que, após a germinação e o estabelecimento de plantas de uma mesma espécie, e estas estando sob a mesma influência ambiental (climática e edáfica), como nesse experimento controlado em casa de vegetação, ocorre uma tendência à homogeneização dos caracteres morfológicos da espécie (VASCONCELOS et al., 2015).

Assim, o efeito de diferentes métodos de superação de dormência sobre o desenvolvimento e estabelecimento inicial morfológico de Annona squamosa L., mostrou-se significativo apenas para tamanho de plantas, o que evidencia que os métodos adotados para a superação de dormência facilitam e aceleram a germinação do embrião no interior da semente, possibilitando o maior crescimento dos indivíduos, pois os mesmos tiveram mais tempo de exposição aos fatores climáticos, e a eficiência de seu aparato fotossintético, o que permite o maior crescimento (SHIMIZU et al., 2011).

\section{CONCLUSÃO}

O tratamento pré-germinativo escarificação mecânica em sementes de Annona squamosa L. (pinha) é o método mais indicado dentre os procedimentos, pois proporciona maior tamanho dos indivíduos após 60 dias.

\section{REFERÊNCIAS}

ATAÍDE, G. M.; BICALHO, E. M.; DIAS, D. C. F. S.; CASTRO, V. O.; ALVARENGA, E. M. Superação de dormência das sementes de Delonix regia (Bojer ex Hook) Raf. Revista Árvore, Viçosa, v. 37, n. 6, p. 1145-1152, 2013. Disponível em: <http://www.redalyc.org/articulo.oa?id=48829909016>.

AZEVEDO, C. F.; QUIRINO, Z. G. M.; REGO, E. R.; BRUNO, R. L. A.; SILVA, G. Z. Anatomical aspects of the Foeniculum vulgare Mill. seedling. Revista Brasileira de Plantas Medicinais, v. 14, p. 197-204, 2012. Disponível em: < http://dx.doi.org/10.1590/S1516-05722012000500013>.

BAGATIM, A, G.; NACATA, G.; ANDRADE, R. A. Efeito de tratamentos para quebra de dormência das sementes na emergência de gravioleira. Interciência, v. 41, n. 9, p. 629-632, 2016. Disponível em:< https://search.proquest.com/docview/1819944471 ?accountid=146829>. 
BARBOSA, Z.; SOARES, I.; CRISÓSTOMO, L. A. Crescimento e absorção de nutrientes por mudas de gravioleira. Revista Brasileira de Fruticultura, v. 25, n. 3, p. 519-522, 2003.

BATAGLION, G. A.; SILVA, F. M. A.; EBERLIN, M. N.; KOOLEN, H. H. F. Determination of the phenolic composition from Brazilian tropical fruits by UHPLCMS/MS. Food Chemistry, v. 180, p. 280-287, 2015. Disponível em:< https://doi.org/10.1016/j.foodchem.2015.02.059>.

doi: 10.1016/j.foodchem.2015.02.059

BEZERRA, P. F. E. C.; PORFÍRIO G. I.; BARROS, T. S.; PEREIRA B. C. Superação de dormência em sementes de Pithecellobium dulce (Roxb.) Benth. Semina: Ciências Agrárias, 2015.Disponínel em:< http://www.redalyc.org/articulo.oa? id=445744146044> doi: 10.5433/1679-0359.2015v36n1p165

CAMPOS, L. F. C.; ABREU, C. M. D.; GUIMARÃES, R. N.; SELEGUINI, A. Scarification and gibberellic acid on emergence and seedling growth of Biriba. Ciência Rural, Santa Maria-RS v. 45, n. 10, p. 1748-1754, 2015.

FERREIRA, G.; ERIG, P. R.; MORO E. Uso de ácido giberélico em sementes de fruta-do-conde (Annona squamosa L.) visando à produção de mudas em diferentes embalagens. Revista Brasileira de Fruticultura, n. 24, n.1, p.178-182, 2002.

FERREIRA, G.; GUIMARÃES, V. F.; PINHO, S. Z. D.; OLIVEIRA, M. C. D.; RICHART, A.; BRAGA, J. F.; DIAS, G. B. Curva de absorção de água em sementes de atemoia (Annona cherimola Mill. x Annona squamosa L.) cv. Gefner. Revista Brasileira de Fruticultura, p. 121-124, 2006. Disponível em: < http://dx.doi.org/10.1590/S0100-29452006000100033>.

FREITAS, A. R.; LOPES, J. C.; MATHEUS, M. T.; MENGARDA, L. H. G.; VENANCIO, L. P.; CALDEIRA, M. V. W. Superação da dormência de sementes de jatobá. Pesquisa Florestal Brasileira, v. 33, n. 73, p. 85-90, 2013. Disponível em: <https://doi.org/10.4336/2013.pfb.33.73.350>. dói: 10.4336/2013.pfb.33.73.350

LEMOS, E. E. P. A produção de anonáceas do Brasil. Revista Brasileira de Fruticultura, v. 36, p. 77-85, 2014.

LOPES, P. S. N.; MAGALHÃES, H. M.; GOMES, J. G.; JÚNIOR, B.; SILVA, D.; ARAÚJO, V. D. D. Superação da dormência de sementes de umbuzeiro (Spondias tuberosa, Arr. Câm.) utilizando diferentes métodos. Revista Brasileira de Fruticultura, 2009. Disponível em: <http://revistas.abaagroecologia.org.br/index.php/cad/article/view/2482>.

MAYER, A. M; POLJAKOFF-MAYBER, A. The Germination of Seeds: Pergamon International Library of Science, Technology, Engineering and Social Studies. Elsevier, 1982.

NETO, A. C. A.; ARAÚJO, P. C.; MOREIRA, E. S. Germinação e vigor de sementes de pinha (Annona squamosa L.) de diferentes procedências. Enciclopédia Biosfera, v. 9, n. 17, p. 1548-1555, 2013. 
NETO, A. F.; DANTAS, B. F.; ALMEIDA, F. A. C.; LIMA, M. S.; SILVA, F. F. S. Influência da embalagem e do local de armazenamento na qualidade fisiológica de sementes de abóbora 'jacarezinho' (Curcubita moschata Duch). Revista Engenharia na Agricultura, Viçosa-MG, v. 22, n. 4, p. 294- 305, 2014.

PIMENTA, A. C.; ZUFFELLATO-RIBAS, K. C.; LAVIOLA, B. G. Morfologia de frutos, sementes e plântulas de Jatropha curcas. Floresta, v. 44, n. 1, p. 73-80, 2013. Disponível em: http://dx.doi.org>. doi:10.5380/rf.v44i1.30878.

PIVETA, G.; MUNIZ, M. F. B.; REINIGER, L. R. S.; DUTRA, C. B.; PACHECO, C. Qualidade sanitária e fisiológica de sementes de aroeira-preta (Lithraea molleoides) submetidas a métodos de superação de dormência. Ciência Florestal, Santa Maria, RS, v. 24, n. 2, p. 289-297, 2014

RAVEN, H. P.; EVERT, R. F.; EICHHORN, S. E. Biologia Vegetal. Editora Guanabara Koogan: Rio de Janeiro, 2014, 876p.

RESENDE, M. D.V. DE Genética biométrica e estatística no melhoramento de plantas perenes. Brasília: Embrapa Informação Tecnológica, 2002. 975p.

SÁNCHEZ, E.G. Polinización artificial del chirimoyo. Granada: Caja rural de Granada, noviembre 1991. 15p.

SANTOS, J. F.; LUZ, I. S.; MATSUMOTO, S. N.; D'ARÊDE, L. O; VIANA, A. E. S. Superação da dormência tegumentar de sementes de Piptadenia viridiflora (Kunth) benth pela escarificação química. Bioscience Journal, v. 30, n. 6, p. 1642-1651, 2014.

SHIMIZU, E. S. C.; PINHEIRO, H. A.; COSTA, M. A.; SANTOS FILHO, B. G. D. Aspectos fisiológicos da germinação e da qualidade de plântulas de Schizolobium amazonicum em resposta à escarificação das sementes em lixa e água quente. Revista Árvore, v. 35, n. 4, p. 791-800, 2011. Disponível em: $<$ http://www.redalyc.org/articulo.oa?id=48819944004.

SILVA, F. A. Z.; AZEVEDO, C. A. V. The Assistat Software Version 7.7 and its use in the analysis of experimental data. Afr. J. Agric. Res, 11(39): 3733-3740, 2016. DOI: 10.5897/AJAR2016.11522.

SILVA, M. D. J. E. Aspectos fisiológicos da dormência em sementes. UniCEUB, 2003.

STENZEL, N. M. C.; MURATA, I. M.; NEVES, C. S. V. J. Superação da dormência em sementes de atemóia e fruta-do-conde. Revista Brasileira de Fruticultura, v. 25, n. 2, p. 305-308, 2003.

TAIZ, L.; ZEIGER, E. Fisiologia vegetal. 5. ed. Porto Alegre: ArtMed, 2013. 954p.

VASCONCELOS, L. H. C.; VENDRUSCULO, E. P.; VASCONCELOS, R. F.; SANTOS, M. M.; SELEGUINI, A. Utilização de métodos físicos e de fitorreguladores 
para superação de dormência em sementes de pinha. Revista de Agricultura Neotropical, v. 2, n. 4, p. 20-24, 2015.

VIEIRA, R. D.; CARVALHO, N. M. Teste de vigor em sementes. Jaboticabal: FUNEP, p. 218, 1994.

ZAR, J. H. Biostatistical analysis. Pearson: Upper Saddle River, p. 944, 2010. 